appeared in Physical Review Letters 104, 058301 (2010)

\title{
Long-time dynamics of concentrated charge-stabilized colloids
}

\author{
Peter Holmqvist ${ }^{1, *}$ and Gerhard Nägele ${ }^{1}$ \\ ${ }^{1}$ Institut für Festkörperforschung, Forschungszentrum Jülich, D-52425 Jülich, Germany
}

\begin{abstract}
Dynamic light scattering was used to study the dynamic structure factor, $S(q, t)$, of suspensions of charged colloidal silica spheres over the full colloidal time range. We show that a dynamic scaling relation for $S(q, t)$ found by Segrè and Pusey (PRL 77, 771 (1996)) for hard spheres, relating longtime and short-time dynamics, and collective and self-diffusion, applies also to charged colloids up to the freezing concentration. The universality of this scaling is analyzed theoretically. Our experimental data confirm dynamic freezing criteria proposed for the long-time self- and cage diffusion coefficients, and a theoretical prediction for the self-diffusion coefficient.
\end{abstract}

PACS numbers: 82.70.Dd, 66.10.cg, 67.10.Jn 
The dynamics in suspension of charged colloidal particles undergoing correlated Brownian motion is of fundamental interest and the subject of ongoing experimental and theoretical research [1-7]. A rich variety of charge-stabilized colloids are encountered in chemical industry, biology and food science. While the static properties of colloids resemble that of atomic liquids, their dynamics is diffusive rather than ballistic. From the theoretical viewpoint this poses a challenging many-body problem where both direct electrosteric and solvent-mediated hydrodynamic interactions (HIs) need to be considered.

Information on the dynamics of colloidal particles is embodied in the dynamic structure factor, $S(q, t)$, which describes the dynamics of spatial Fourier components of thermal fluctuations in the number density as a function of correlation time $t$ and scattering wavenumber $q$. It can be determined by dynamic light scattering (DLS) $[1,8]$ and X-ray photon correlation spectroscopy $[4,6]$. From the knowledge of $S(q, t)$ in the colloidal time range, a deeper insight into transport properties such as diffusion coefficients and viscoelastic quantities [9] is obtained, in the fluid state and at crystallisation [10] and vitrification points [11]. Short-time dynamic properties of charged colloids are now rather well understood thanks to detailed scattering experiments [12] and elaborate computer simulations [13]. In contrast to this, a lot remains to be learned about the long-time dynamics.

A comprehensive study on the dynamics of suspensions of neutral hard spheres was made by Segrè and Pusey using two-color DLS [14]. They measured the $S(q, t)$ of hard spheres in the whole fluid concentration regime, for the full span of correlation times. The key finding of their study is that the normalized dynamic structure factor, $f(q, t)=S(q, t) / S(q)$, of hard spheres of radius $a$ is well approximated for $q>2.5 / a$ including the principal peak of $S(q)$, by the scaling relation $[14]$

$$
f(q, t) \approx \exp \left\{-q^{2} \frac{D_{S}(q)}{d_{S}} W(t)\right\} .
$$

Here $W(t)=\left\langle\Delta r^{2}(t)\right\rangle / 6$ is the mean-square displacement of a particle with initial and longtime slopes equal to, respectively, the short-time and long-time self-diffusion coefficients $d_{S}$ and $d_{L}$. Furthermore, $D_{S}(q)=d_{0} H(q) / S(q)$ is the short-time diffusion function proportional to the hydrodynamic function, $H(q)$, which reflects the influence of HIs. The single-particle diffusion coefficient is denoted by $d_{0}$. The Segrè-Pusey factorization suggests that density relaxations at non-small wavenumbers are controlled by self-diffusion.

Eq. (1) assumes that the $q$ and $t$ dependence of $\log f(q, t)$ can be factorized. It reproduces 
the limiting small-q and large-q behavior of $f(q, t)$, namely $f\left(q \ll q_{m}, t\right) \approx \exp \left\{-q^{2} D_{S}(q) t\right\}$ and $f\left(q \gg q_{m}, t\right) \propto \exp \left\{-q^{2} W(t)\right\}$. Here, $q_{m} \approx 3.5 / a$ is the locus of the principal peak of the hard-sphere $S(q)$. The large- $q$ form is valid for negligible non-Gaussian corrections, important in particular near a glass transition point. Measuring $f(q, t)$ for $q>2.5 / a$ should give a $q$-independent master master curve equal to $W(t) / d_{S} t$, when $-\ln f(q, t) /\left(q^{2} D_{S}(q) t\right)$ is plotted versus $t$. Moreover, a single-exponential decay, $f(q, t) \propto \exp \left\{-q^{2} D_{L}(q) t\right\}$, is predicted for long times and $q>2.5 / a$, with a long-time diffusion function

$$
\frac{D_{L}(q)}{D_{S}(q)} \approx \frac{d_{L}}{d_{S}}
$$

of same $q$-dependence as $D_{S}(q)$, and ratio $d_{L} / d_{S}$. Note that $D_{L}(q \rightarrow \infty)=d_{L}$ since $D_{S}(q \rightarrow \infty)=d_{S}$. An empirical rule for the onset of freezing by Löwen et al. [10] states that $d_{L} / d_{S} \approx 0.1$ at freezing. Thus the $D_{L}(q)$ of a concentrated system is substantially smaller than its short-time counterpart.

The key question is whether Eqs. (1) and (2) are generic features of colloidal dispersions, not restricted to the system of polymethacrylate (PMMA) hard-spheres in cis-decalin studied by Segrè and Pusey. A recent study has challenged the validity of Eq. (2) even for the restricted class of hard-sphere-like systems: Using XPCS and small-angle X-ray scattering (SAXS), Lurio et al. [15] measured the diffusion of charge-stabilized polystyrene latex spheres in glycerol. The static structure factor $S(q)$ in their system is indistinguishable from that of hard spheres, but the ratio $D_{L}(q) / D_{S}(q)$ was found to vary strongly in $q$ at higher volume fractions $\phi$, in conflict with Eq. (2). Lurio et al. argue that the violation of Eq. (2) may be due to different hydrodynamic boundary conditions on the surface of a charge-stabilized latex and a sterically stabilized PMMA sphere.

In this letter, we describe DLS experiments on fluid-ordered, charge-stabilized trimethoxysilypropyl methacrylate (TPM) coated silica spheres [16], dispersed in an indexmatching 80/20 toluene-ethanol solvent mixture at $T=20^{\circ} \mathrm{C}$. The particle radius determined by SAXS is $a=136 \mathrm{~nm}$, with a small size polydispersity of 0.06 . The residual salinity in the system is below $1 \mu \mathrm{M}$. Our precision measurements of $S(q, t)$ cover the full colloidal time regime and a broad range of wavenumbers, with $\phi$ extending up to the freezing transition value $\phi_{f} \approx 0.16$ where $S\left(q_{m}\right) \approx 3.2$. The DLS measurements were made using a light scattering set-up by the ALV-Laservertriebsgesellschaft (Langen, Germany). The intensity autocorrelation function, $g_{2}(q, t)$, was recorded by an ALV-5000 multi-tau digital correlator, 


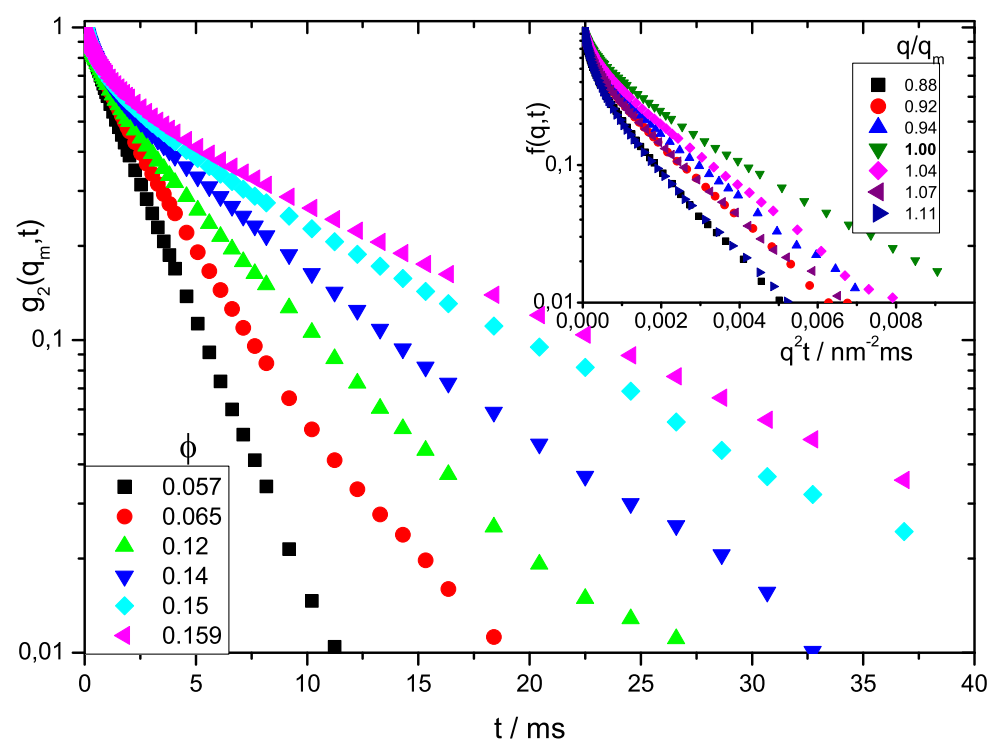

FIG. 1: Scattering function $g_{2}(q, t)$ versus time $t$ for TPM coated silica spheres in a toluene-ethanol mixture measured at $q=q_{m}(\phi)$, for $\phi$ values as indicated. Inset: $f(q, t)$ versus $q^{2} t$ for $\phi=0.14$, at $q$ values as indicated.

and the electric field autocorrelation function $g_{1}(q, t) \propto S(q, t)$ was determined from $g_{2}(q, t)$ using the Siegert relation. We have checked that there is no noticeable multiple scattering in our system.

We will show that our results for $D_{S}(q)$ and $d_{S}$ deduced from $S(q, t)$ are in good agreement with theoretical predictions for low-salt systems of strongly repulsive charged particles $[13,17,18]$. Moreover, our data at the crystallization point confirm two dynamic criteria proposed, respectively, for the freezing values of $d_{L} / d_{S}$ [10] and the long-time cage diffusion coefficient, $D_{L}\left(q_{m}\right)$ [18]. The latter quantifies the decay of density relaxations of wavelength $2 \pi / q_{m}$, comparable in size to the dynamic cage of neighbors. Most importantly, our data show that the factorization scaling applies approximately also to charge-stabilized suspensions for concentrations up to the freezing point. We provide theoretical arguments supporting the applicability of factorization scaling for arbitrary pair potentials, but we also point to its approximate nature for intermediate and long times.

Fig. 1 shows DLS data for the $g_{2}(q, t)$ of TPM coated silica spheres at $q=q_{m}$, and for $\phi$ up to the freezing value. The inset displays $\ln f(q, t)$ versus $q^{2} t$, for $\phi=0.14$ and wavenumbers 
including the peak position value $q_{m}$ where $S\left(q_{m}\right) \approx 2.9$. The experimental $S(q)$ is shown in the inset of Fig. 2, together with the $S(q)$ calculated using the penetrating background rescaled mean spherical approximation (PBRMSA) scheme [19]. This analytic scheme gives accurate structure factors, as shown recently by in an extensive comparison with simulation data, and results from the similarly accurate but numerically far more costly Rogers-Young scheme [20]. The only adjustable parameter in this scheme is the effective particle charge in the Derjaguin-Landau-Verwey-Overbeek (DLVO) screened Coulomb potential, determined as 175 e from fitting the experimental peak height. The good agreement between calculated and experimental $S(q)$ holds for all investigated concentrations. According to Fig. $1, f(q, t)$ and $g_{2}(q, t)$ of strongly correlated particles decay initially exponentially, followed by a nonexponential decay at intermediate times, and an approximately exponential slower decay at long times. Like for the PMMA suspension studied earlier [14, 21], we have deduced the long-time diffusion function $D_{L}(q)$ from these data. The inverse of $D_{L}(q)$ in Fig. 2 is compared with the inverse of $D_{S}(q)$ plotted on a different vertical scale. In accord with Eq. (2), $D_{L}(q)$ and $D_{S}(q)$ share practically the same $q$-dependence, with ratio $D_{L}(q) / D_{S}(q) \approx 0.23$ at $\phi=0.14$. This super-positioning is valid for $\phi$ up to the freezing value. The solid curve in Fig. 2 is the prediction for $D_{S}(q)$ by the $\delta \gamma$ scheme of Beenakker and Mazur [22] using the PBRMSA structure factor shown in the inset as input. The agreement with the experimental data illustrates the accuracy of this scheme in predicting the $H(q)$ of charge-stabilized systems $[12,13]$.

To test Eq. (1) for its time dependence, in Fig. 3 we plot $-\ln f(q, t) /\left(q^{2} D_{S}(q) t\right)$ versus $t$, for the same $q$ values as in the inset of Fig. 1. To decent accuracy, the data collapse on a master curve for $q>0.8 q_{m}$. According to Eq. (1), this curve should be identified as $W(t) /\left(d_{S} t\right)$ which has the long-time asymptote $d_{L} / d_{S}$. The long-time regime is reached approximately for $t>33 \mathrm{~ms}$, giving an estimate of $d_{L} / d_{S}(\phi=0.14) \approx 0.23$.

A discussion is in order here on the validity of the factorization scaling, and the existence of the exponential long-time mode in $f(q, t)$ as its necessary prerequisite. The occurrence of a long-time mode and its $q$ dependence were explored theoretically by Cichocki and Felderhof [23, 24], for dilute to moderately dense hard-sphere systems without HIs. They analyzed the spectral distribution function, $p_{q}(\lambda) \geq 0$, of relaxation modes,

$$
f(q, t)=\int_{0}^{\infty} d \lambda p_{q}(\lambda) \exp \{-\lambda t\}
$$




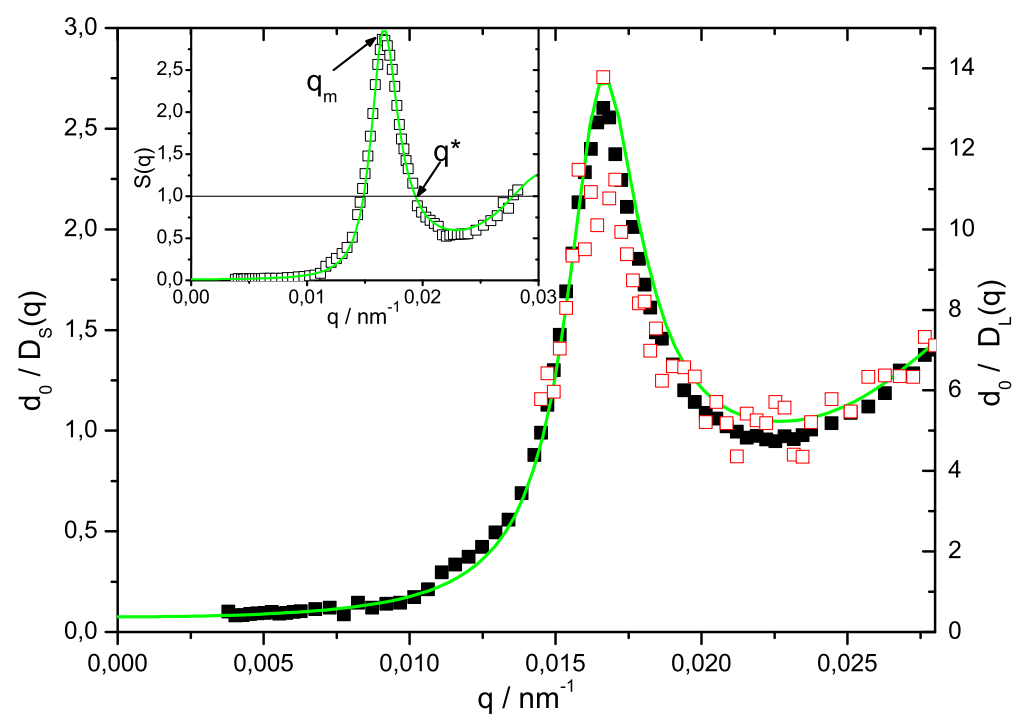

FIG. 2: DLS results for the normalized inverse short-time diffusion function $d_{0} / D_{S}(q)$ (filled squares, scale on left axis) and inverse long-time diffusion function $d_{0} / D_{L}(q)$ (open squares, scale on right axis) of TPM coated silica spheres at $\phi=0.14$. Inset: static structure factor. Lines: theoretical predictions for $d_{0} / D_{S}(q)$ by $\delta \gamma$ scheme with PBRMSA input for $S(q)$.

describing the strictly monotonous decay of $f(q, t)$, for its dependence on the relaxation rates $\lambda$. Using a contact Enskog approximation (CEA) exact to first order in $\phi$, they showed that for $S(q)$ sufficiently large at the considered $q$, the long-time decay of $f(q, t)$ is dominated indeed by an exponential long-time mode. This mode arises from an isolated delta peak contribution to $p_{q}(\lambda)$, located in a low $\lambda$ gap, where the continuous part of $p_{q}(\lambda)$ is zero. As explained in $[23,24]$, the associated coefficient $D_{L}(q)$ relates to the center of mass diffusion of a pair of particles. At a wavenumber different from $q_{m}$ where $S(q)$ is small, e.g., near to the first minimum in $S(q)$ to the right of $q_{m}$, the delta peak can mold with the continuous part of the spectrum, but still some pronounced peak in $p_{q}(\lambda)$ may remain. If sufficiently strong, this peak describes approximately a long-time exponential mode. Quite interestingly, the ratio $D_{L}(q) / D_{S}(q)$ obtained in CEA using that $D_{S}(q)=d_{0} / S(q)$ for zero HIs, varies significantly as a function of $q[24]$.

The feature of a long-time mode for hard spheres without HIs, observed for $\phi \geq 0.2$ [25] where $S\left(q_{m}\right)$ is sufficiently large, should survive to a decent approximation when particles 


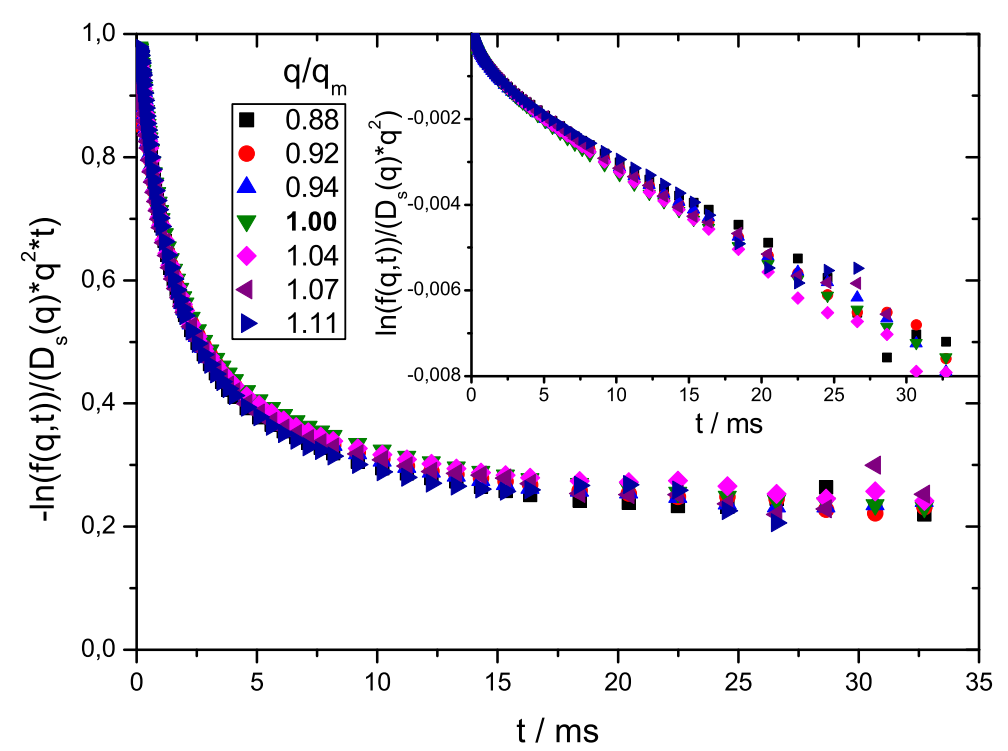

FIG. 3: Same data as in the inset of Fig. 1, plotted here as $-\ln (f(q, t)) /\left(q^{2} D_{S}(q) t\right)$. Inset: $\ln (f(q, t)) /\left(q^{2} D_{S}(q)\right)$ versus $t$. Scaling is found for $q / q_{m} \geq 0.8$.

with soft interactions, and many-body correlation effects not accounted for in the CEA are considered. Indeed, mode coupling theory (MCT) calculations without HIs predict a longtime mode both for charge-stabilized spheres with strong electrostatic interactions, and dense hard-sphere systems [25]. Again, no sharp long-time mode occurs when the system is too weakly structured, and when $S(q)$ at the considered $q$ is too small (see also Fig. 1).

We expect that HIs cause no qualitative changes. However, the earlier experimental findings for neutral PMMA spheres and our present data for charged silica spheres suggest that HIs tend to smooth out the $q$-variations in $D_{L}(q) / D_{S}(q)$ that would be present otherwise. The existence of an (approximate) long-time mode with $D_{L}(q)$ proportional to $D_{S}(q)$ does not necessarily imply validity of the $q-t$ factorization in $\ln f(q, t)$. To check the validity of Eq. (1) for a dense hard-sphere system, we performed MCT calculations of $W(t)$ and $f(q, t)$, each shown to be in good agreement with Brownian Dynamics simulation results [26]. According to MCT, the time dependence in Eq. (1) given by $W(t)$ is only an approximation, especially at intermediate times. There is no reason to expect Eq. (1) to become exact in the presence of HIs.

Since the self-intermediate scattering function, $G(q, t)$, related to self-diffusion is not mea- 
surable by DLS, we use an approximate procedure by Pusey to extract $d_{S}$ and $d_{L}$ directly from $S(q, t)$ [27], measured at a wavenumber $q^{*}>q_{m}$ (see inset of Fig. 2) where the distinct structure factor, $S_{d}(q)=S(q)-1$, is zero. On assuming that the time-dependent generalization, $S_{d}\left(q^{*}, t\right)=S\left(q^{*}, t\right)-G\left(q^{*}, t\right)$, remains small as compared to $G\left(q^{*}, t\right)$, it follows $S\left(q^{*}, t\right) \approx G\left(q^{*}, t\right)$, and consequently $S\left(q^{*}, t\right) \approx \exp \left\{-\left(q^{*}\right)^{2} W(t)\right\}$ when non-Gaussian contributions are ignored. This identifies $d_{S}$ with $D_{S}\left(q^{*}\right)$. Computer simulations of neutral and charged colloidal spheres [13], show that $d_{S}$ is determined by this procedure to within 5 $10 \%$ of accuracy. A similar degree of accuracy can be expected for $d_{L}$. This is supported by MCT results for charged particle systems where we compare $-\ln S\left(q^{*}, t\right) /\left(q^{*}\right)^{2}$ to $W(t)$, observing an overall agreement within 10\% deviations at intermediate times [26]. Pusey's procedure to determine $W(t)$ is consistent with factorization scaling, as one notices from substituting $D_{S}\left(q^{*}\right)$ in Eq. (1) by $d_{S}$. Our experimental data confirm the theoretical short-time prediction, $d_{s} / d_{0}=1-2.5 \phi^{4 / 3}[13,25]$, for all considered $\phi$ values. The $\phi^{4 / 3}$-dependence is

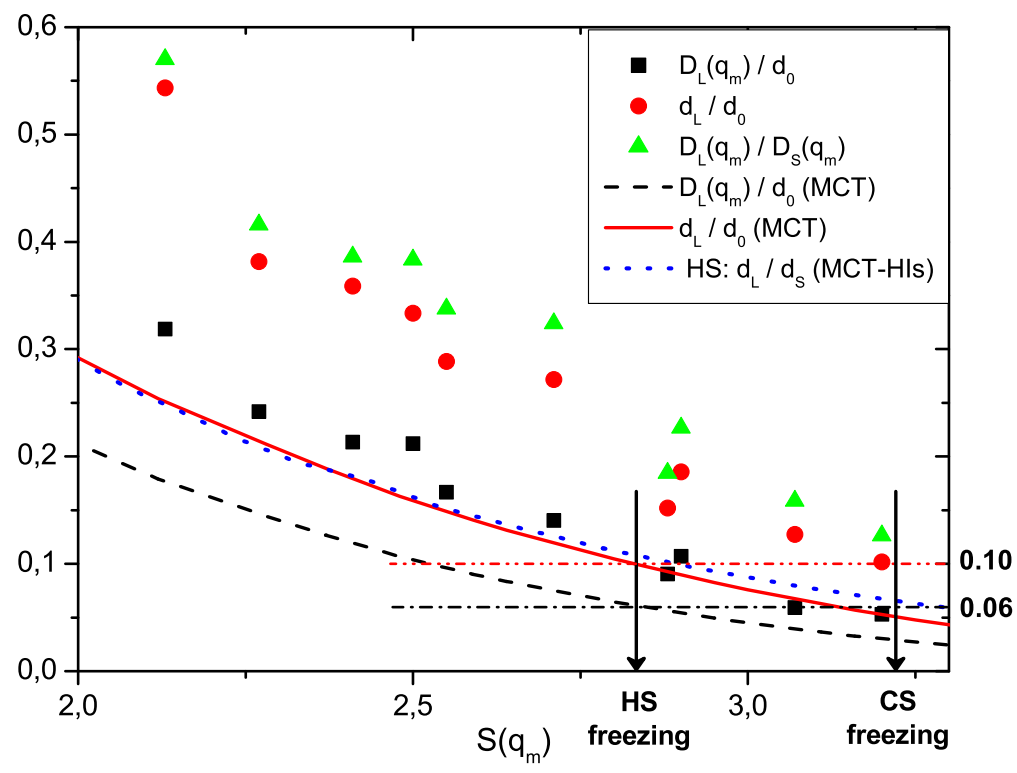

FIG. 4: Long-time cage diffusion coefficient $D_{L}\left(q_{m}\right) / d_{0}$ and self-diffusion coefficient $d_{L} / d_{0}$, and selfdiffusion ratio $d_{L} / d_{S}$ versus $S\left(q_{m}\right)$. Filled symbols: experimental data for charged silica spheres. Lines: MCT prediction for charged spheres (CS) without HIs (denoted by MCT), and HIs-corrected MCT predictions for hard spheres (HS) denoted by MCT-HIs. 
characteristic of charged-particle systems at low salinity where $q_{m} \propto \phi^{1 / 3}$.

The long-time self- and cage diffusion coefficients decrease in general monotonically with increasing $\phi$, to an extent depending on interaction parameters such as salinity and particle charge. For a discussion of their generic behavior, our data for $d_{L} / d_{0}, D_{L}\left(q_{m}\right) / d_{0}$ and $D_{L}\left(q_{m}\right) / D_{S}\left(q_{m}\right) \approx d_{L} / d_{s}$ are plotted in Fig. 4 as a function of $S\left(q_{m}\right)$, and compared to theoretical predictions. Note that $S\left(q_{m}\right)$ quantifies the overall strength of static correlations. The MCT curve for the self-diffusion ratio, $d_{L} / d_{S}$, of hard spheres has been corrected for HIs by a short-time hydrodynamic rescaling procedure [2, 25]. It describes experimental data very well [18]. At the freezing value $S\left(q_{m}, \phi_{f}=0.494\right)=2.85$ of hard spheres, it gives $d_{L} / d_{S} \approx 0.1$ in accord with Löwen's criterion. While the short-time rescaling procedure works well for hard spheres [2], it does not apply to low-salinity systems of charged particles, where opposite to hard spheres long-time diffusion is enhanced [3, 18, 28].

The MCT curve for $d_{L} / d_{0}$ of charged spheres has been obtained without HIs. It matches rather closely the $d_{L} / d_{S}$ curve for hard spheres, giving $d_{L} / d_{0} \approx 0.1$ at $S\left(q_{m}\right)=2.85$, and $d_{L} / d_{0} \approx 0.06$ at the experimental value $S\left(q_{m}\right)=3.2$ where the silica system freezes. The freezing value for $S\left(q_{m}\right)$ varies, depending on the range and strength of the pair potential, from 2.85 for hard spheres to about $3.1-3.3$ for low-salt suspensions with long-range electrostatic repulsions $[29,30]$. From the silica system data, we note $d_{L} / d_{S} \approx D_{L}\left(q_{m}\right) / D_{S}\left(q_{m}\right) \approx$ 0.12 at freezing, in decent agreement with Löwen's criterion. Another remarkable finding is that the experimental freezing value, $D_{L}\left(q_{m}\right) / d_{0} \approx 0.05$, for the cage diffusion coefficient is close to the value 0.06 predicted by MCT at the peak height were hard spheres freeze. At the experimental peak height of the silica system at freezing, MCT predicts $D_{L}\left(q_{m}\right) / d_{0} \approx 0.03$.

As shown in $[18,25]$, a universal 1-1 relation between $d_{L} / d_{0}$ and $S\left(q_{m}\right)$, and a corresponding relation between $D_{L}\left(q_{m}\right) / d_{0}$ and $S\left(q_{m}\right)$, should apply approximately to all wellstructured, low-salt systems characterized by a single length scale $r_{m} \approx \rho^{-1 / 3} \propto 1 / q_{m}$, where $\rho$ is the particle number density. With HIs, radius a comes into play as a second physically relevant length so that $d_{L} / d_{0}$ and $D_{L}\left(q_{m}\right) / d_{0}$ become moderately dependent on the ratio $r_{m} / a$ [18]. Inclusion of HIs has the effect to move the theoretical curves for $d_{L} / d_{0}$ and $D_{L}\left(q_{m}\right) / d_{0}$ closer to the experimental values, which otherwise are larger roughly by a factor of 2. The reason for the upward shift caused by the HIs is that, according to theory and simulations $[3,18,28], d_{L}$ is enlarged by the far-field part of the HIs dominating in lowsalinity systems, by a factor of about $1.15-1.35$. Since $D_{L}\left(q_{m}\right) \sim d_{L}$, a similarly pronounced 
hydrodynamic enforcement can be expected for $D_{L}\left(q_{m}\right)$, but there are no simulation data available to date to corroborate this. According to our experiments, $d_{S}$ of low-salt systems is smaller than $d_{0}$ by a factor of $1-2.5 \phi^{4 / 3}$, so that $d_{L}$ is further upshifted when normalized by $d_{S}$ in lieu of $d_{0}$. However, one should not expect a quantitative agreement with the experimental data even after the inclusion of HIs effects. MCT is an approximate approach after all, which becomes increasingly less accurate for less strongly structured systems. In addition, one should not disregard the unavoidable scatter in the long-time experimental data.

In summary, we have shown that the factorization scaling of $f(q, t)$, with $q$-independent ratio $D_{L}(q) / D_{S}(q)$ for $q>0.8 q_{m}$, applies also to charge-stabilized suspensions. According to our theoretical discussion, this approximate feature should be observable, to varying degree of accuracy, in any suspension of rigid spherical colloidal particles, provided the particles are correlated sufficiently strongly. In addition, our experimental data confirm theoretical predictions for the freezing values of $d_{L} / d_{S}$ and $D_{L}\left(q_{m}\right)$, the $\phi$-dependence of $d_{S}$ and the $q$-dependence of $D_{S}(q)$. We expect dynamic scaling to apply approximately even in case of suspensions of soft spherical colloidal particles such as microgel spheres [31] and certain core-shell particles [32], where dynamic differences to rigid particles arise mainly from the solvent permeability and the correspondingly weakened HIs. A strong interplay of the intraand inter-particle dynamics can be expected for very soft and flexible particles such as the soft giant micelles studied in [33], which might severely affect dynamic scaling. To explore this interplay will require more theoretical and experimental work.

We thank M. Heinen and J. Gapinski for help with the short-time calculations and data evaluation, and A. Banchio and B. Chichocki for helpful discussions. This work and the printing of the article was under appropriation of funds from the Deutsche Forschungsgemeinschaft (SFB-TR6, project B2).

* Electronic address: p.holmqvist@fz-juelich.de

[1] P.N. Pusey, in Liquids, Freezing and the Glass Transition, edited by J.P. Hansen, D. Levesque and J. Zinn-Justin (Elsevier, Amsterdam, 1991).

[2] A.J. Banchio, J. Bergenholtz and G. Nägele, Phys. Rev. Lett. 82, 1792 (1999). 
[3] W. Härtl et al., J. Phys.: Condens: Matter 12, A287 (2000).

[4] D.O. Riese et al., Phys. Rev. Lett. 85, 5460 (2000).

[5] L.F. Rojas et al., Faraday Discuss 123, 385 (2003).

[6] A.J. Banchio et al., Phys. Rev. Lett. 96, 138303 (2006).

[7] C. Haro-Pérez et al., Phys. Rev. Lett. 102, 018301 (2009).

[8] W. Härtl, H. Versmold, U. Wittig and P. Linse, J. Chem. Phys. 97, 7797 (1992).

[9] A.J. Banchio, G. Nägele and J. Bergenholtz, J. Chem. Phys. 111, 8721 (1999).

[10] H. Löwen, T. Palberg, and R.G. Simon, Phys. Rev. Lett. 70, 1557 (1993).

[11] Ch. Beck, W. Härtl and J. Wagner, J. Chem. Phys. 111, 8209 (2000).

[12] J. Gapinski et al., J. Chem. Phys. 130, 084503 (2009).

[13] A.J. Banchio and G. Nägele, J. Chem. Phys. 128, 104903 (2008).

[14] P.N. Segrè and P.N. Pusey, Phys. Rev. Lett. 77, 771 (1996).

[15] L.B- Lurio et al., Phys. Rev. Lett. 84, 785 (2000).

[16] A. P. Philipse and A. Vrij, J. Chem. Phys. 88, 6459 (1988).

[17] G. Nägele, Phys. Rep. 272, 216 (1996).

[18] G. Nägele, M. Kollmann, R. Pesché and A.J. Banchio, Molec. Phys. 100, 2921 (2002).

[19] I.K. Snook and J.B. Hayter, Langmuir 8, 2280 (1992).

[20] M. Heinen, A.J. Banchio and G. Nägele, to be submitted.

[21] P.N. Segrè, S.P. Meeker, P.N. Pusey and W.C.K. Poon, Phys. Rev. Lett. 74, 1250 (1995).

[22] C.W.J. Beenakker and P. Mazur, Physica 126 A, 349 (1984).

[23] B. Cichocki and B.U. Felderhof, J. Chem. Phys. 98, 8186 (1993).

[24] B. Cichocki and B.U. Felderhof, Physica A 204, 152 (1994).

[25] A.J. Banchio, G. Nägele and J. Bergenholtz, J. Chem. Phys. 113, 3381 (2000).

[26] A.J. Banchio, P. Holmqvist and G. Nägele, unpublished results.

[27] P.N. Pusey, J. Phys. A 11, 119 (1978).

[28] P. Baur and G. Nägele, Europhys. Lett. 38, 557 (1997).

[29] J.-P. Hansen and L. Verlet, Phys. Rev. 184, 151 (1969).

[30] K. Kremer, M.O. Robbins and G.S. Grest, Phys. Rev. Lett. 57, 2694 (1986).

[31] T. Eckert and W. Richtering, J. Chem. Phys. 129, 124902 (2008).

[32] G. Petekidis et al., Phys. Rev. E 69, 042401 (2004).

[33] R. Sigel et al., Phys. Rev. Lett. 83, 4666 (1999). 